\title{
NEW DYNAMIC CHANNEL ALLOCATION ALGORITHMS
}

\author{
Ailton A. Shinoda and Michel D. Yacoub \\ Grupo de Pesquisa em Comunicações - CODEC \\ Depto. Desenv. sistemas - CPqD - TELEBRÁS \\ CP 1579 - Campinas - SP. CEP 13088-061 \\ fone : (019)705742 \\ Depto. de Comunicações - FEEC - UNICAMP \\ CP 6101 - Campinas - SP - CEP 13081-970 \\ fone : (019)7883812 - fax : (019) 2891395 \\ michel@decom.fee.unicamp.br
}

Resumo -Um conjunto de três algoritmos de alocação de canais é proposto e analisado. Ao contrário de muitos algoritmos, cuja performance depende grandemente do perfil de tráfego, as técricas propostas adaptam-se dinarnicamente às mudanças da carga de tráfego, combinando as características da alocação đinâmica de canais com as de alocação fixa de canais, migrando suavemente de uma para outra técnica para dar a melhor performance em quaisquer circunstâncias. Embora os algoritmos propostos alocam os canais de maneira totalmente dinâmica, isto é feito dentro de uma dada disciplina de forma que os canais sejam empacotados em grupos de reuso e a distância de reuso é mantida em um mínimo, aumentando a eficiência de reuso.

Abstract - A set of three allocation algorithms is proposed and analysed. As opposed to a number of allocation algorithms, whose performance is greatly dependent on the traffic profile, these techniques are dynamically adaptable to the change of the traffic load, combining the features of dynamic channel allocation and fixed channel allocation, migrating smoothly from one to another technique to give the best performance in any circumstances. Although the proposed strategies assign the channels in a fully dynamic fashion, this is carried out in a disciplined way so that channels are packed into reuse groups and the reuse distance is kept to a minimum, increasing the reuse efficiency.

Keywords :wireless communications, channel allocation, traffic performance.

\section{INTRODUCTION}

Efficient use of radio resources has always represented a major concem in wireless communications due to the scarcity of the frequency spectrum. A substantial effort has been spent towards investigating techniques that lead to a better system performance with a direct impact on capacity. Channel allocation is certainly one of such techniques.

A great many chamel allocation algorithms have been proposed and extensively explored in the literature (e.g. [1-4]). Essentially, these algorithms can be grouped into Fixed Channel Allocation (FCA), in which the channels are assigned on a rigid way according to a given traffic distribution and a given grade of service, and Dynamic Channel Allocation (DCA), in which an arbitrary proportion of the channels is assigned on a demand basis. The performance of these techniques is greatly dependent on the traffic distribution and, in general, DCA performs less satisfactorily than FCA for high load [5-8]. In fact, in DCA, the continuous change of the usage pattern easily leads to spatial inefficiency because of the channel locking, a technique used to keep the cochannel interference within acceptable limits by impeding the channels to be reused unless the required minimum reuse distance is satisfied. If this is not appropriately handled, as the traffic grows the number of locked channels also grows and the DCA techniques are outperformed by the FCA one, where the channels are packed into reuse groups and the reuse distance is kept to a minimum.

This paper proposes three channel allocation algorithms dynamically adaptable to the change of the traffic load. They conveniently combine the features of FCA and DCA, migrating smoothly from one to another technique to always give the best performance in any circumstances.

\section{THE TECHNIQUES}

The aim of the techniques to be proposed here is to assign the channels dynamically but on an orderly and compact basis such that the channel locking is minimised. Because of the disciplined usage of the dynamic channels, as the traffic grows, the system evolves gradually and smoothly to the fixed allocation pattern, combining, then, the benefits of DCA for low traffic and of FCA for high traffic.

Before proceeding to the description of the techniques we explore the terminology to be used and the basic procedures to be assumed in a dynamic allocation. Define :

- $I(v)$ as the set of the cells interfering with cell $\mathrm{v}$

- $D(v)$ as the set of cells with the minimum reuse distance with respect to cell $\mathrm{v}$

- $A_{i}$ as the set of cells where channel $i$ has been assigned

- $v_{c}$ as the cell where a new call arises

In order to maintain the cochannel interference within the specification limits, the minimum requirement for a channel $i$ to be assigned to a call arising in cell $v_{a}$ is the fulfilment of the basic condition $v_{\alpha} \notin A_{i}$ and $I\left(v_{\alpha}\right) \cap A_{i}$ 
$=\varnothing$, where $\varnothing$ denotes the null set. The new call will be blocked if $v_{a} \notin A_{i}$ or $I\left(v_{a}\right) \cap A_{i} \neq \emptyset \vee i \in N$, where $N$ is the set of channels within the system. The algorithm choosing the first channel satisfying this basic condition is the well-known First Available (FA) algorithm, the simplest form of DCA. Some of the allocation techniques proposed here include the FA as part of their overall decision procedure. It must be emphasised, however, that many other forms of DCA, as available in the literature, could be used, instead.

The next subsections describe 1) the procedure common to the three proposed techniques, 2) the proposed techniques, named $\alpha$ Algorithm, $\beta$ Algorithm, and $\gamma$ Algorithm, and 3) the call reshuffle process.

\subsection{Common Procedure}

In a procedure common to all of the proposed algorithms, the sets $Z_{i}\left(v_{a}\right)=A_{i} \cap D\left(v_{a}\right) \vee i \in N$ are built and a channel is chosen that satisfies the condition $\max \left|Z_{i}\left(v_{a}\right)\right| \vee i \in N$, where the module representation $|X|$ signifies the number of elements within the set $X$. The chosen channel is then assigned to a new call.

Note that $Z_{i}\left(v_{a}\right)$ gives the cocells of cell $v_{a}$ using channel $i$ i at the minimum reuse distance and that $\max \left|Z_{i}\left(v_{a}\right)\right|$ gives the channel with the highest usage in the nearest cocells at the time of the assignment procedure.

\section{2. $\alpha$-Algorithm}

This algorithm follows the common procedure whenever $Z_{i}\left(v_{a}\right) \neq \emptyset$. However, when $Z_{i}\left(v_{a}\right)=\emptyset \vee i \in N$ and $B_{m}\left(v_{a}\right) \leq G O S$, where $B_{m}\left(v_{a}\right)$ is the mean blocking probability of $v_{a}$ and $G O S$ is the specified grade of service, a channel $j$ is assigned that satisfies the basic condition $v_{a} \notin A_{i}$ and $I\left(v_{a}\right) \cap A_{i}=\emptyset$. In case $Z_{i}\left(v_{a}\right)=\emptyset \vee i \in N$ and $B_{m}\left(v_{a}\right)>G O S$ but $A_{k} \neq \emptyset$ such a call will be blocked even if a chamel n exists that satisfies $v_{a} \notin A_{n}$ and $I\left(v_{a}\right) \cap A_{n}=\emptyset$.

In essence, in this algorithm a new call is assigned the channel with the highest usage in the nearest cocells at the time of the assignment procedure. In case no channel is found that satisfies such a condition and the mean blocking probability of the cell satisfies the specified grade of service the first available channel is allotted. If the mean blocking probability exceeds the required grade of service, a channel is selected among those not being used. If this fails, the call is blocked.

\section{3. $\beta$-Algorithm}

This algorithm follows the common procedure whenever $Z_{i}\left(v_{a}\right) \neq \emptyset$. However, when $Z_{i}\left(v_{a}\right)=\emptyset \vee i \in N$ and $L\left(v_{a}\right) / N \leq(r-l) / r$, where $L\left(v_{a}\right)$ is the number of locked chamels in $v_{a}$ and $r$ is the chosen reuse pattern, a channel $j$ is assigned that satisfies the basic condition $v_{a} \notin A_{i}$ and $I\left(v_{a}\right) \cap A_{i}=\emptyset$. In case $Z_{i}\left(v_{a}\right)=\emptyset \vee i \in N$ and $L\left(v_{a}\right) / N>(r-l) / r$, a a channel $\mathrm{k}$ is assigned that satisfies the condition $A_{k}=\emptyset$. If $Z_{i}\left(v_{a}\right)=\emptyset \vee i \in N$ and $L\left(v_{a}\right) / \dot{N}>(r-l) / r$ but $A_{k} \neq \emptyset$ such a call will be blocked 46 even if a channel $n$ exists that satisfies the basic condition $v_{a} \notin A_{n}$ and $I\left(v_{a}\right) \cap A_{n}=\emptyset$.

The motivation to choose the ratio $(r-l) / r$ as a decision point is that, when FCA is used with a given reuse pattern $r$ say $r=7$, i.e. 7 cells per cluster) only a proportion $l / r(1 / 7$, for $r=7$ ) of all the channels can be used within the cell; the $(r-1) / r(6 / 7$, for $r=7)$ ) remaining proportion represents that of the locked channels.

In essence, in this algorithm a new call is assigned the channel with the highest usage in the nearest cocells at the time of the assignment procedure. In case no channel is found that satisfies such a condition and the proportion of locked channels of the cell is less or equal than the corresponding proportion for the chosen reuse patten the first available channel is allotted. If the proportion of locked channels of the cell exceeds that of the chosen reuse pattern, a channel is selected among those not being used. If this fails, the call is blocked.

\section{4. $\gamma$-Algorithm}

This algorithm follows the common procedure whenever $Z_{i}\left(v_{a}\right) \neq \emptyset$. However, when $Z_{i}\left(v_{a}\right)=\emptyset \vee i \in N$, a channel $j$ is assigned that satisfies the condition $A_{j}=\emptyset$. The call will be blocked in $v_{a}$ if $Z_{i}\left(v_{a}\right)=\emptyset$ and $A_{i} \neq \emptyset \vee i, j \in N$, even if a chamnel $k$ exist that satisfies $v_{a} \notin A_{k}$ and $I\left(v_{a}\right) \cap A_{k}=\emptyset$.

In essence, in this algorithm a new call is assigned the channel with the highest usage in the nearest cocells at the time of the assignment procedure. In case no channel is found that satisfies such a condition, then a channel is selected among those not being used. If this fails, the call is blocked. Therefore, this algorithm keeps a rigid control of the reuse distance which is constantly maintained to its minimum

\subsection{Call Reshuffle}

At the end of each call a call reshuffle procedure takes place aiming at increasing the number of active cocells using the same channel at the minimum reuse distance.

Suppose a call using channel $j$ in cell $v_{a}$ has ended and assume that $k$ is the channel satisfying the condition $\min \left|Z_{i}\left(v_{a}\right)\right| \vee i \in N$, where $Z_{i}\left(v_{a}\right)=A_{i} \cap D\left(v_{a}\right)$. A reshuffle from the call using channel $k$ to the released channel $j$ will take place if the number of cocells at the minimum reuse distance using channel $j$ is bigger than that of cocells at the minimum reuse distance using channel $k$.

\section{SYSTEM UNDER INVESTIGA- TION}

The performance of the proposed techniques has been assessed in several cellular network configurations with distinct traffic distributions[9] In particular, the results to be shown here consider the traffic to be uniformly distributed over an "infinite" system of 49 cells displaced on a toroidal surface, as shown in Figure 1. The parameters used as decision criteria in the algorithms are $G O S=5 \%$ and $r=7$, with clusters 
having 105 channels. Traffic is considered to be Poisson, holding time has a negative exponential distribution with mean equal to 2 minutes, and subscribers are assumed to be fixed. A Monte Carlo simulation has been used to assess the performance, given in terms of the system mean blocking probability.

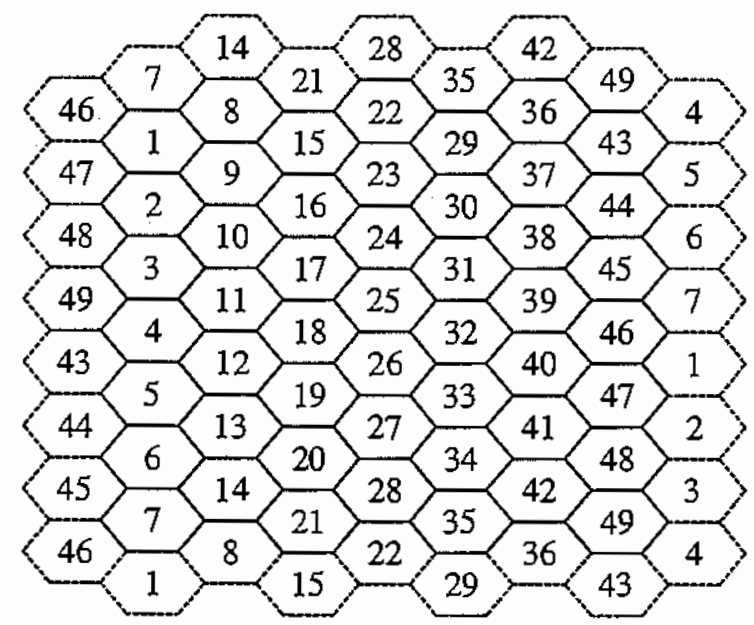

FIG. 1: Infinite cellular layout.

\section{RESULTS}

Broadly speaking, among the three proposed algorithms, there are two distinct strategies, namely those carried out in the $\alpha$ and in the $\beta$ Algorithms and those carried out in the $\gamma$ Algorithm. The $\gamma$ Algorithm is included in the other two and it is accomplished in the $\alpha$ Algorithm when the mean blocking probability of the cell exceeds that of the required grade of service $(G O S)$, chosen as a decision parameter. In the same way, it is accomplished in the $\beta$ Algorithm when the proportion of locked channels of the cell (a function of the reuse pattern) exceeds that of the specified value for the chosen reuse pattern. In fact the performance of the $\alpha$ Algorithm will approach that of the $\gamma$ Algorithm as the GOS, chosen as a threshold, tends to $0 \%$. The same reasoning applies to the $\beta$ Algorithm with the allowed proportion of locked channels. These threshold points can be set as required and this is a useful mechanism that can be exercised to check the simulation programs.

Figure 2 shows the system mean blocking probability as a function of the traffic load per cell normalised with respect to 8 erl.. Because some of the allocation algorithms proposed here (namely $\alpha$ and $\beta$ ) include the FA technique as part of their overall decision procedure we also compare their performances with that of FA. As mentioned before, it must be emphasised, however, that many other forms of DCA, as available in the literature, could be used, instead of FA. Figure 2 also shows the performance of FCA for comparison.

Note that, as quoted previously, FA performs better than FCA for low traffic but it is outperformed by FCA with high load. And this behaviour is noticed in a number of DCA techniques [5-8]. Note also that all of the three algorithms proposed here perform substantially better than FCA and FA both

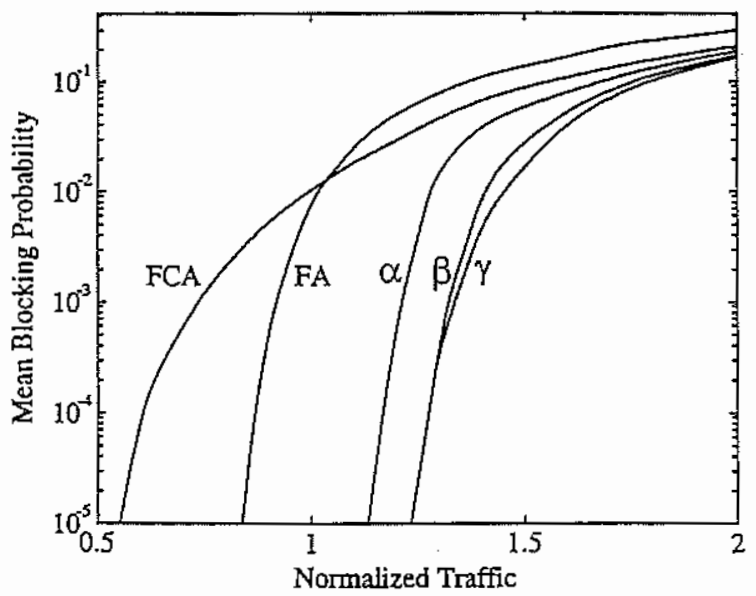

FIG. 2: Mean blocking probability versus traffic normalised with respect to 8 erl. per cell.

for low traffic and for high load. It is interesting to note that all of the three algorithms lead the system asymptotically to the FCA performance with the increasing traffic. In the limit as the traffic tends to infinity the performance of the proposed algorithms coincides with that of the FCA. In other words, although the proposed techniques assign the channels in a fully dynamic fashion, this is carried out in a disciplined way so that channels are packed into reuse groups and the reuse distance is kept to a miniroum, as in FCA.

It can be seen that among the proposed techniques the $\gamma$ Algorithm gives the best performance, followed by the $\beta$ Algorithm, and the $\alpha$ Algorithm in this order. For a $2 \%$ system mean blocking probability, for instance, the traffic gain with respect to FCA is $35 \%, 32 \%$, and $16 \%$ for the respective algorithms. (Note that in such a case FA is already outperformed by FCA.) The traffic gain is noticeably higher than this in an unbalanced traffic system [9].It is clear, from these results, that a flexibility in assigning the channels must be allowed and, at the same time, a careful control of the channel locking condition is mandatory in order for the system to adapt itself to the variation of the traffic load to give the best performance. An interesting point to be mentioned is that the best strategy, performed by the $\gamma$ Algorithm, presents decision rules that are rather simple to implement and are completely independent of any threshold parameter.

\section{CONCLUSIONS}

A set of three dynamic channel allocation algorithms have been proposed and analysed. A Monte Carlo simulation study has been carried out having an "infinite" cellular network with uniform traffic distribution as the system under investigation. In general, these techniques maximise the number of cochannels already in use and minimise the number of channels in a locked condition.

As opposed to a number of allocation algorithms, whose performance is greatly dependent on the traffic, these techniques are dynamically adaptable to the change of the traffic load. They combine the features of dynamic channel alloca- 
tion and fixed channel allocation conveniently, and a smooth migration from one to another technique occurs so that the best performance is accomplished in any circumstances. Although the proposed techniques assign the channels in a fully dynamic fashion, this is carried out in a disciplined way so that channels are packed into reuse groups and the reuse distance is kept to a minimum, increasing the reuse efficiency. Therefore, the techniques require, as an input parameter, among others, the target reuse pattern, which is chosen to fulfil the interference specifications. In theory, if in the course of the system operation a new reuse pattern is found that suits the network more adequately, the algorithm can then be updated with the new input, and the new reuse pattern shall be targeted. (In practice, things are certainly much more intricate.)

The proposed algorithms are rather simple and have their decision rules based on the number of active cochannels, the specified grade of service, and the allowed proportion of locked channels. It is noteworthy that the best strategy, performed by $\gamma$ Algorithm, is independent of any threshold parameter and bases its decisions on the target reuse pattern.

These techniques have been simulated in several network configurations, with different traffic distributions, and it has been observed that the qualitative results shown here are also applicable to these systems.

\section{REFERENCES}

[1] M. Zhang and T. S. Yum, "Comparison of Channel Assignment Strategies in Cellular Mobile Telephone Systems," IEEE Transactions on Vehicular Technology, Vol. 38, no. 4, November 1989, pp. 211-215.

[2] I. Katzela and M. Naghshineh, "Channel Assignment Schemes for Cellular Mobile Telecommunication Systems," A Comprehensive Survey, IEEE Personal Communications, Vol. 3, no. 3, June 1996, pp. 10-31.

[3] M. D. Yacoub and K. W. Cattermole, "Altemative routing strategies for adjacent cells in mobile radio systems," IEE Proc.-Commun., Vol. 142, No. 2, pp. 115-120, April 1995.

[4] A. A. Shinoda and M. D. Yacoub, "Combined techniques for channel allocation algorithms in mobile radio systems," IEE Proc.-Commun., Vol. 144, No. 3, pp. 205-210, June 1997.

[5] D. C. Cox and D. O. Reudink: "Increasing channel occupancy in large-scale mobile radio systems: Dynamic channel reassignment," IEEE Trans. on Commun., Vol. COM-21, No. 11, Nov. 1973, pp. 1302-1306.

[6] J. S. Engel and M. M. Peritsky: "Statistically-optimum dynamic server assignment in systems with interfering servers", IEEE Trans. Veh. Technol., Vol. VT-22, No. 4, pp. 203-209, Nov. 1973.

[7] L. G. Anderson: "A simulation study of some dynamic assignment algorithm in a high capacity mobile telecommunication system," IEEE Trans. on Comm, Vol. COM21, No. 11, Nov. 1973, pp. 1294-1301.
[8] S. M. Elnoubi, R. Singh, and S. C. Gupta: "A new frequency channel assignment algorithm in high capacity mobile communication systems", IEEE Trans. Veh. Technol., Vol. VT-31, No. 3, pp. 125-131, Aug. 1982.

[9] A. A. Shinoda, "Channel allocation algorithms in wireless communications systems," $\mathrm{PhD}$ Thesis, University of Campinas, 1996.

Ailton Akira Shinoda- received the B.Sc. (Eng.), the M.Sc. (Eng.) and Ph.D. (Eng.) from the University of Campinas (UNICAMP) in 1986, 1993 and 1996, respectively. During 1997-1998 he was a Visiting Researcher in the Division of Electrical and Computer Engineering, Yokohama National University. Since 1986 he has been working as a researcher at the R\&D Center of Telebras (now CPqD Fundation), where his research interests concentrate in mobile communications such as traffic simulation, channel allocation, W-CDMA simulation.

Michel Daoud Yacoub was born in Brazil in 1955. He received the B.S.E.E and the M.Sc. degrees from the School of Electrical and Computer Engineering of the State University of Campinas, UNICAMP, Brazil, respectively in 1978 and 1983, and the Ph.D. degree from the University of Essex, England, in 1988. From 1978 to 1985, he worked as a research specialist at the R\&D Center of Telebrás, Brazil, in the development ofthe Tropico digital exchange family. He joined the School of Electrical and Computer Engineering, UNICAMP, in 1989, where he is presently an associate professor. He has already served as Head of the Communications Department at the same School. He consults for several operating companies and industries in the wireless communications area. He is the author of the book Foundations of Mobile Radio Engineering, CRC Press, 1993, and the co-author of the book Telecommunications: Principl es and Trends (in Portuguese), Erica Press, 1997. He authored chapters in The Mobile Communications Handbook, CRC Press and IEEE Press, 1996, The Communications Handbook, CRC Press and IEEE Press, 1996, The Electronics Handbook, CRC Press and IEEE Press, 1996, and in The Mobile Communications Handbook (2nd edition), CRC Press and IEEE Press, USA, 1998. He holds two patents and has published more than 60 technical papers in highly reputable journals and conferences. He has also published more than 60 articles in a specialized Brazilian telecommunications newspaper. His book Foundations of Mobile Radio Engineering, CRC Press, 1993, already in its 7th reprint, is considered to be a best seller and has been adopted as textbook for wireless communications courses in more than 20 Universities all over the world. His other book (Telecommunications Principles and Trends, Erica Press, 1997), in its 2nd edition, has been used as text in several multidisciplinary courses in several Brazilian Universities. He has supervised (already concluded) more than twenty Ph.D. and M.Sc. Theses. His research interests include wireless communications in general. 